\title{
Metabolic Marker
}

National Cancer Institute

\section{Source}

National Cancer Institute. Metabolic Marker. NCI Thesaurus. Code C18520.

Specific metabolic changes that characterize an organism's state of health or disease, or response to a particular therapeutic intervention, and helps make diagnosis more accurate or enable physicians to make diagnosis before symptoms appear and to track disease progression. 